\title{
The Influence of the Levonorgestrel-Releassing Intrauterine System (Lng Ius) on Metabolic Markers in Women with Normal Body Mass and Overweight Women
}

\author{
Ineta Vasaraudze*,**, Dace Rezeberga**,***, Renars Erts**, Aivars Lejnieks**,*** \\ *I.Vasaraudze Private Clinic Ltd, Riga, Latvia \\ **Riga Stradins University, Latvia \\ $* * *$ Riga East Clinical University Hospital, Riga, Latvia
}

\begin{abstract}
Summary
Introduction. In Latvia, the number of overweight women is increasing, while the rate of cardio vascular disease (CVD) is one of the highest in the European Union. The influence of hormonal contraception on the development of CVD has not been studied in Latvia so far.

Aim of the study. A nonrandomized open-label prospective trial of healthy reproductive-age women desiring to use the LNG IUS. Materials and methods. Before starting the use of contraception and six months after starting the use of contraception, the homeostasis model assessment insulin resistant (HOMA-IR) score, insulin sensitivity (HOMA-\%S) and $\beta$-cell function (HOMA-\%B) were calculated using fasting glucose $(\mathrm{FG})$ and $\mathrm{C}$ peptide values. There were also determined other cardio-metabolic parameters: total cholesterol, high density lipoprotein cholesterol, low density lipoprotein cholesterol, triglyceride level, systolic and diastolic blood pressure, and abdominal circumference.

Results. Thirty women were involved in the study: seventeen women with a normal body-mass index (BMI) (BMI below 25) and thirteen overweight women (BMI above 25). When using the LNG IUS, the fasting glucose level increased in women with normal body mass, the level of insulin sensitivity decreased in both study groups; $C$ peptide level, $\beta$-cell function and insulin resistance increased in both groups, but the changes were not statistically reliable $(p>0.05)$.

Conclusion. Although during the study there were determined changes in the FG level, insulin sensitivity, C peptide, $\beta$-cell function and insulin resistance parameters, these changes are not clinically significant, and the LNG IUS may be safely used clinically both by women with normal body mass and overweight women.
\end{abstract}

Keywords: Levonorgestrel-releasing intrauterine system, contraception, body mass index, insulin resistance, fasting glucose.

\section{INTRODUCTION}

In Latvia, cardio vascular disease (CVD) claims more female lives than cancer and accidents combined. In 2006, CVD caused the death of $73 \%$ of Latvian women (58\% men). CVD related death-rate is higher and the average life expectancy of patients is lower than in most of the European countries(7).

With the increase of diabetes, obesity and metabolic syndrome, CVD will continue to rise. An estimated $10 \%$ of the population in Latvia are obese (BMI of 30 or higher), an astounding $30 \%$ of women have a BMI of 25 or higher(7). The distribution of obese persons varies between age groups. The share of obese persons in the age group of $25-44$ year is $9.6 \%$, but in the age group of $45-64$ year $-31 \%$ (3). For women there is a clear pattern: the incidence of obese persons increases with age. Compared to the women with normal body mass, obese women have a higher risk of hypertension, type 2 diabetes and the development of metabolic syndrome. The American Heart Association has identified the following major CVD risk factors: hypertension, hyperlipidemia, diabetes and several risk factors unique to women like gestational diabetes, pregnancy-inducted hypertension \& preeclampsia, polycystic ovarian syndrome (6). Since pregnancy-related complications are more common among obese women than among women with normal body weight, the choice of a safe long-term contraception method is of extreme importance.

Long-acting reversible contraceptive methods (LARC) include: the copper containing intrauterine device (IUD), the etonogestrel containing sub-dermal implant (not available in Latvia yet) and the levonorgestrelreleasing intrauterine system (LNG IUS). Both methods available in Latvia are long acting (5-12 years), very effective (Perl index is 0.8 and 0.2 for the typical use in one year, respectively), and there is no evidence of a delay in the return of fertility following the removal of IUDs or LNG IUS.

According to the report "The Reproductive Health of the Population (2003-2011)", when choosing contraception, almost a half of the women surveyed mentioned such criteria as the beneficial effect on the quality of life and 
simple usage as important. Overall, the inhabitants are well informed about the usage of contraceptive methods; nevertheless, safe protection methods are not chosen or are used wrongly, which implies that the respondents are not able to use their knowledge in practice in an effective way. However, only one respondent in ten indicated a safe long-term contraceptive method as an important criterion (1).

The LNG IUS has been approved for contraception and heavy menstrual bleeding therapy in Latvia since 1998. The use of the LNG IUS is increasing, particularly in the treatment of endometriosis, endometrial hyperplasia (8).

Since 2006 the LNG IUS has been included in the list of reimbursable drugs in Latvia with the indication N92 excessive, frequent and irregular menstruation ( ICD10). Each year about 1500 LNG IUS are administered to patients having this condition. About $90 \%$ of these patients are in the age group of 30-55; about $60 \%$ belong to the age group of 40-55 (7).

Contrary to other progestogen-only contraceptives, the activity of LNG IUS is based on a local effect on the endometrium and non-essential systemic effects. Taking into account the high proportion of overweight and obese patients and the increasing number of LNG IUS users in Latvia, the effects of the LNG IUS on metabolic markers - serum lipid levels, fasting glucose level and insulin resistance - are of considerable interest. The effects on various metabolic indicators have been studied in young women using LNG IUS for contraception (5) and in women suffering from heavy menstrual bleeding. Poor patient compliance to this treatment is common as patients are afraid of systemic effects caused by hormonal preparations, mainly the weight gain. The Homeostasis Model Assessment (HOMA) estimates steady state beta cell function (\%B) and insulin sensitivity (\%S), as percentages of a normal reference population and has become the method of choice for evaluating changes in metabolic markers. HOMA uses fasting glucose (FG) and $\mathrm{C}$ peptide measurements to calculate homeostasis assessment model insulin resistance (HOMA-IR), insulin sensitivity (HOMA-\%S), and $\beta$-cell function (HOMA-\%B).

The effect of these methods of hormonal contraception on the metabolic parameters of women has not been studied in Latvia so far. The purpose of the study was to clarify the cardio-metabolic characteristics of LNG IUS users and the possible impact of the LNG IUS on these parameters.

\section{AIM OF THE STUDY}

A nonrandomized open-label prospective trial of healthy reproductive-age women desiring to use the LNG IUS.

\section{MATERIALS AND METHODS}

\subsection{Subjects}

During a 24-month period (January 2011 - January 2013), women who wanted to have LNG IUS inserted as a contraceptive in I. Vasaraudze Private Clinic Ltd were invited to participate in an open-label prospective trial of healthy reproductive-age women desiring to use LNG IUS. All the potential participants of the study had to undergo screening in order to exclude diabetes (defined by American Diabetes Association criteria) (6), dislipidemia, CVD, the clinical polycystic ovarian syndrome (PCOS) diagnosis (with oligomenorrhea and hirsutism) or other contra-indications for the use of LNG IUS. The exclusion criteria were as follows: unwillingness to undergo the examinations included in the study, a period of less than 6 months after the delivery, less than three months after the use of another hormonal contraceptive, a planning pregnancy in the nearest 12 months. It was intended to recruit up to 30 women willing to use the LNG IUS or to recruit those who came first until the 24-month recruitment period ended. Women received an electronic invitation letter; no payments or gifts were offered for participation. The patients paid for all the examinations. Prior to the study an informed patient consent was obtained to use the clinical information gathered for statistical analysis and publication. This study was approved by the Ethics Committee of Riga Stradins University

\subsection{Procedures}

Once the enrollment visit was completed, laboratory examinations were conducted on starting the use of the LNG IUS (on the day of insertion) and six months after the placement of the contraceptive system. During each visit, 8-12 hours after the last meal, there was performed venopuncture in order to determine the level of fasting glucose and $\mathrm{C}$ peptide. The examination plan also included: determining the BMI, the measurement of arterial blood pressure and abdominal circumference (AC); besides, a single fasting blood sample was taken to determine total cholesterol (TC), high density lipoprotein cholesterol (HDLC), low density lipoprotein cholesterol (LDLC), and triglyceride levels. The LNG IUS was inserted on 1-7 day of the menstrual cycle after collecting the blood samples and performing the clinical examinations. Six months after the insertion of the LNG IUS, the patients were invited to come for a repeated visit in order to perform repeated FG, C peptide and lipid metabolism tests in a blood sample, as well as do the measurements of abdominal circumference, blood pressure and body mass.

\subsection{Study drugs}

According to the LNG IUS product description, Mirena ${ }^{\circledR}$ (Bayer Schering Pharma Oy, Tuku, Finland) is a type-T intrauterine system (IUS) that releases the hormone levonorgestrel after its insertion in the uterus, 20 micrograms in 24 hours. The stable hormone concentration in the blood at $150-200 \mathrm{pg} / \mathrm{mL}$ is achieved a few weeks after the insertion of the system.

3.4. Laboratory and bio-analytical methods

The body mass was measured for all women without footwear using the Terraillon ${ }^{\circledR}$ TPRO 3100 balance (Terraillon UK Ltd, Hermel Hempsted, UK). The height was measured for all women without footwear with the stadiometer MPS-A06 (Kern\&Sohn GmbH, Balingen, Germany). The weight was approximated to the nearest $0.1 \mathrm{~kg}$, the height - to $0.1 \mathrm{~cm}$. AC was measured at the 
level of the navel. The blood pressure was measured with the sphygmomanometer Riester (Rudolf Riester GmbH, Jungingen, Germany) after sitting for 5 and 10 minutes: the mean figure between two measurements was used for the analysis of the data.

Fasting biochemical analyses were collected and kept in the room temperature until their delivery to E. Gulbis Laboratory Ltd (Riga, Latvia) using special courier equipment for the transportation of medical analyses. $\mathrm{C}$ peptide was analyzed using the immunochemical luminescence method with the analyzer Immulite 2000 (Siemens Healthcare Diagnostics). LDLC, HDLC, TC and triglycerides were analyzed automatically with the clinical chemical analyzer Integra 400, (Roche Diagnostics). Glucose was analyzed automatically with the clinical chemical analyzer Hitachi 917 (Roche Diagnostics).

The HOMA Calculator $\odot$ The University of Oxford 2004 (www.OCDEM.ox.ac.uk) was used to determine HOMA-IR, HOMA-\%S, HOMA-\%B online using the measurements of $\mathrm{FG}$ and $\mathrm{C}$ peptide.

The patients were divided into groups depending on their BMI. The following subdivision (according to the international obesity taskforce - IOTF) was used: underweight: less than 18.5; normal weight: between 18.5 and less than 25; overweight: between 25 and less than 30; obese: equal or more than 30.

3.5. Data analysis

Microsoft Excel was used in creating the database and producing graphs, while the data were analyzed using the Statistical Package for the Social Sciences (SPSS) version 17 for Windows. The commonly used $p<0.05$ rule was applied to detect significant differences.

\section{RESULTS}

In the period of 1 January 2011 and 31 December 2012, 48 women were screened for the participation in the study; 30 women corresponded to all the inclusion criteria and agreed to participate in the study (Figure 1). Among 30 women who chose the LNG IUS as a contraceptive and were recruited for the present study, 17 had a normal BMI and 13 had a BMI above 25. The group of women with the BMI above 25 included 2 women with the BMI above 30, while the number of the participants having adiposity was too small to make a separate sub-group, and these women were included in the group of overweight women. The demographic characteristics and the initial baseline examinations of the study participants are reflected in Table 1. At the beginning of the study, no significant differences were singled out between the groups concerning the arterial blood pressure, FG, C peptide, LDLC or any of the three HOMA indexes(IR, \%S un \%B). However, there were statistically significant differences between the groups in the abdominal circumference measurements $(\mathrm{p}=0.001)$; on average, it was above $10.45 \mathrm{~cm}$ higer in the BMI $>25$ group; HDLC level $(p=0.03)$, on average it was higher than $0.4 \mathrm{mmol} / \mathrm{L}$ in the $\mathrm{BMI}<25$ group, and the average triglyceride level was higher than $0.45 \mathrm{mmol} / \mathrm{L}(\mathrm{p}=0.03)$ in the $\mathrm{BMI}<25$ group.
Six months after using the LNG IUS, no statistically significant differences were recognized in the metabolic markers between the groups. In the BMI $>25$ group, there was found an average weight increase of $1.03 \mathrm{~kg}$ and an average increase in the abdominal circumference of $0.9 \mathrm{~cm}$; on the other hand, in the BMI $<25$ group, there was found an average weight loss of $1.3 \mathrm{~kg}$ and no changes in the abdominal circumference. During the period of using the LNG IUS, the combined cholesterol LDLC, HDLC, TGs levels were stable and unchanged in both groups.

Despite the fact that no statistically significant differences were found between the groups, there was observed a clinically insignificant increase in the FG and $C$ peptide level $(0.23 \mathrm{mmol} / \mathrm{L}$ and $0.89 \mathrm{ng} / \mathrm{mL}$, respectively) in the $\mathrm{BMI}>25$ group. Both study groups showed an increase in HOMA-IR (by 0.5 in the BMI $<25$ group and 0.11 in the BMI $>25$ group, respectively). During the study, there was found a decrease in HOMA-\%S and an increase in HOMA-\%B in both groups. When both time and group effects were calculated in the analysis, there were no significant differences in HOMA-\%B or HOMA-IR between the two groups (Figures 2,3,4,5 and $6)$.

\section{DISCUSSION}

In the present study, there was not noticed any changes in the cardio-metabolic markers during six-month period in LNG IUS users of LNG IUS users irrespective of their BMI when starting the use of the contraceptive. The group of LNG IUS users with a normal BMI at the entry in the study showed an increase in the FG level; there was also determined an increase in body weight and abdominal circumference. It may be that a decrease in \% S caused by levonorgestrel on absorption in insulin sensitive organs (muscles and liver) results in the higher FG level (2).

The situation concerning obesity among the Latvian women of the reproductive age implies that there is an absolute need for studies concerning safe contraception methods in this group. It is very important for these women to use safe contraceptives since pregnancyrelated complications are quite common in this population. Therefore, the contraception methods which do not make the cardio-metabolic morbidity rate worse are a very topical issue. So far, the data concerning the influence of the LNG IUS on lipid metabolism are contradictory. Most of the studies report statistically significant changes in the lipid profile in long-term users (9).

The use of the LNG IUS for the treatment of excessive menstrual bleeding in 48 premenopausal women (the average age 44 years) in the period of 12 months showed a FG increase for 5 women, who initially had a elevated FG level (4); the lack of the control group does not allow to evaluate whether these changes are related to the use of the LNG IUS. Despite the fact that there was a slight increase in the overall FG level among the LNG IUS users, the FG was still at a normal level at the end of the study. 
The present study involved the comparison of metabolic markers between the LNG IUS users with normal BMI and elevated BMI. The limitation of the study is the short duration of the follow-up of this trial. Another limitation is the small number of the study participants; it may be that the small number of the study participants did not make it possible to identify statistically significant changes in HOMA-IR among the IUS users. This study demonstrates that overweight women may have a different metabolic reaction to the use of the LNG IUS. If the situation concerning obesity among the Latvian women of reproductive age continues to growth at the same rate, it may be necessary to consulting these women more and more often about the contraception methods that do not increase the risk of CVD. Despite the fact that the study showed an increase in the FG level and insulin sensitivity (\%S) among the LNG IUS users, the benefits resulting from this contraception method exceed the potential risk of the users of the system.

\section{CONCLUSION}

Although during the study there were determined changes in the FG level, insulin sensitivity, C peptide, $\beta$-cell function and insulin resistance parameters, these changes are not clinically significant, and the LNG IUS may be safely used clinically both by women with normal body mass and overweight women. At present, it is not clear yet whether the identified changes would continue to progress during the long-term use of the LNG IUS and thus might cause long-term health consequences (a higher risk of metabolic syndrome and diabetes) in the users of the system.

\section{Conflict of interest: None}

\section{REFERENCES}

1. Available at: http://papardeszieds.lv/en/index. php? option $=$ com_content $\delta$ view $=$ article\&id $=13$ 4:sexual-and-reproductive-health-research-inlatvia-\&catid=1 1:projects\&Itemid=40. Accessed September 2012

2. Bender NM, Segall-Gutierrez P, Najera SO, et al. Efeects of progestin-only long-acting contraception on metabolic markers in obese women// Contraception, Forthcoming 2013

3. Eurostat Newsrelease 172/2011-24 November 2011, European Health Interview Survey, Between $8 \%$ and $25 \%$ of adults are obese across Member States. Available at: http://epp. eurostat.ec.europa.eu/statistics_explained/index. php/Overweight_and_obesity_-_BMI_statistics and http://epp.eurostat.ec.europa.eu/cache/ITY PUBLIC/3-24112011-BP/EN/3-24112011-BP-EN. PDF 1

4. Kaykicioglu F, Gunes M, Ozdegirmenci O, Haberal A. Effects of the levonorgestrel-releasing hormone system on glucose and lipid metabolism: a 1-year follow-up study// Contraception, 2006; 73:528-31
5. Morin-Papunen L, Martikainen H, McCarthy MI, et al. Comparison of metabolic and inflammatory outcomes in women who used oral contraceptives and the levonorgestrel-releasing intrauterine device in a general population// Am J Ostet Gynecol, 2008; 199:529.1- 10

6. Mosca L, Benjamin E J, Berra K et al. Effectiveness-based guidelines for the prevention of cardiovascular disease in women- 2011 update: a guideline from the American Heart Association// Circulation 123(11), 1243-1262(2011)

7. National health Service. Available at: http://vec. gov.lv/uploads/files/4d00e0402bec2.pdf

8. Varma R, Sinha D, Gupta J. Non- contraceptive uses of levonorgestrel-releasing hormone system(LNG-IUS)- a systematic enquiry and overview// Eur J Obstet Gynecol Reprod Biol, 2006; 125:9-28

9. Woo NY, Liang S, Singh K. Effects of Mirena (levanoregestrel- releasing intrauterine system) and Ortho Gyanae T380 intrauterine copper device on lipid metabolism- a randomized comparative study// Contraception, 2009;79:24-8

\section{Address:}

Ineta Vasaraudze,

A. Čaka str. 92-6b,

Riga, Latvia, LV-1011,

E-mail: ineta@vasaraudze.lv

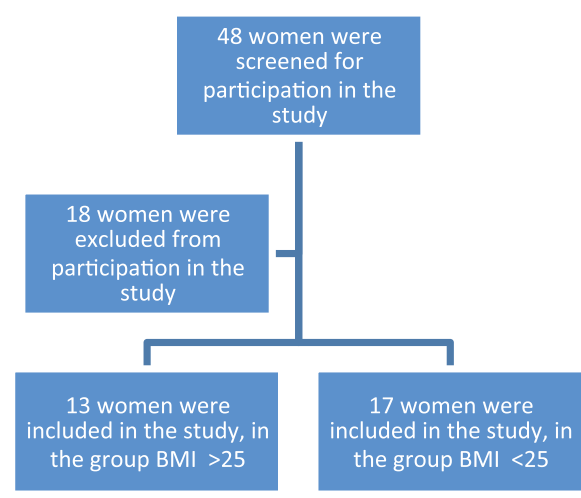

Fig. 1. The study sampling scheme 
Table 1. The comparison of baseline characteristics among study subjects

\begin{tabular}{|c|c|c|c|}
\hline Variable $^{a}$ & $\mathrm{BMI}>25$ & $\mathrm{BMI}<25$ & p value ${ }^{b}$ \\
\hline Age & $36.38(4.57)$ & $34.2(6.66)$ & 0.32 \\
\hline Parity & $1.46(1.84)$ & $1.76(1.24)$ & 0.33 \\
\hline Weight (kg) & $79.18(179.73)$ & $63.04(7.51)$ & $<0.05^{c}$ \\
\hline $\mathrm{AC}(\mathrm{cm})$ & $89.15(9.77)$ & $78.7(6.17)$ & $0.001^{\mathrm{c}}$ \\
\hline SBP (mmHG) & $121.92(15.88)$ & $112.70(13.23)$ & 0.094 \\
\hline DBP (mmHg) & $81.53(14.19)$ & $72.94(10.90)$ & 0.071 \\
\hline HDLC (mmol/L) & $1.59(0.22)$ & $1.70(0.39)$ & $0.03^{c}$ \\
\hline LDLC (mmol/L) & $1.55(0.34)$ & $1.95(0.54)$ & 0.65 \\
\hline $\mathrm{TC}(\mathrm{mmol} / \mathrm{L})$ & $5.11(0.91)$ & $5.23(0.82)$ & 0.72 \\
\hline TGs (mmol/L) & $1.36(0.52)$ & $0.91(0.54)$ & $0.03^{c}$ \\
\hline $\mathrm{FG}(\mathrm{mmol} / \mathrm{L})$ & $4.91(0.47)$ & $4.87(0.53)$ & 0.82 \\
\hline C peptide (ng/mL) & $1.96(0.99)$ & $1.6(0.64)$ & 0.23 \\
\hline IR (HOMA-IR, score) & $1.43(0.74)$ & $1.22(0.37)$ & 0.32 \\
\hline$\beta$-cell function(HOMA-\%B, \%) & $124.24(43.22)$ & $116.68(38.43)$ & 0.61 \\
\hline$\%$ S(HOMA-\%S, \%) & $87.56(44.62)$ & $89.32(29.52)$ & 0.89 \\
\hline
\end{tabular}

a data presented as mean (SD) or as frequency (\%)

${ }^{\mathrm{b}} \mathrm{p}$ value testing for differences in mean or frequencies among the two groups

c Significant at 0.05 level via the chi- square test.

Table 2. The comparison of metabolic markers at 6 months among study subjects

\begin{tabular}{|l|l|l|l|}
\hline Variable $^{\mathrm{a}}$ & BMI $>25$ & $\mathrm{BMI}<25$ & $\mathrm{p}$ value $^{\mathrm{b}}$ \\
\hline Weight $(\mathrm{kg})$ & $77.88(11.75)$ & $64.07(8.93)$ & $<0.01^{\mathrm{c}}$ \\
\hline AC $(\mathrm{cm})$ & $90.46(10.31)$ & $79.64(7.84)$ & $0.003^{\mathrm{c}}$ \\
\hline SBP $(\mathrm{mmHG})$ & $122.3(13.48)$ & $119.41(14.98)$ & 0.145 \\
\hline DBP $(\mathrm{mmHg})$ & $79.23(10.37)$ & $78.23(13.8)$ & 0.83 \\
\hline HDLC (mmol/L) & $1.59(0.22)$ & $1.70(0.39)$ & 0.36 \\
\hline LDLC (mmol/L) & $3.01(0.58)$ & $2.6(0.78)$ & 0.34 \\
\hline TC (mmol/L) & $5.08(0.54)$ & $4.79(1.06)$ & 0.38 \\
\hline TGs (mmol/L) & $1.12(0.44)$ & $0.89(0.32)$ & 0.33 \\
\hline FG (mmol/L) & $4.85(0.62)$ & $5.1(0.51)$ & 0.23 \\
\hline C peptide (ng/mL) & $2.12(1.25)$ & $2.49(3.20$ & 0.69 \\
\hline IR (HOMA-IR, score) & $1.54(0.97)$ & $1.72(1.75)$ & 0.73 \\
\hline$\beta$-cell function (HOMA-\%B, \%) & $137.13(47.91)$ & $121.65(57.56)$ & 0.44 \\
\hline \% S (HOMA-\%S, \%) & $75.81(21.5)$ & $83.85(40.42)$ & 0.52 \\
\hline
\end{tabular}

a data presented as mean (SD) or as frequency (\%)

${ }^{\mathrm{b}} \mathrm{p}$ value testing for differences in mean or frequencies among the two groups

c Significant at 0.05 level via the chi- square test. 

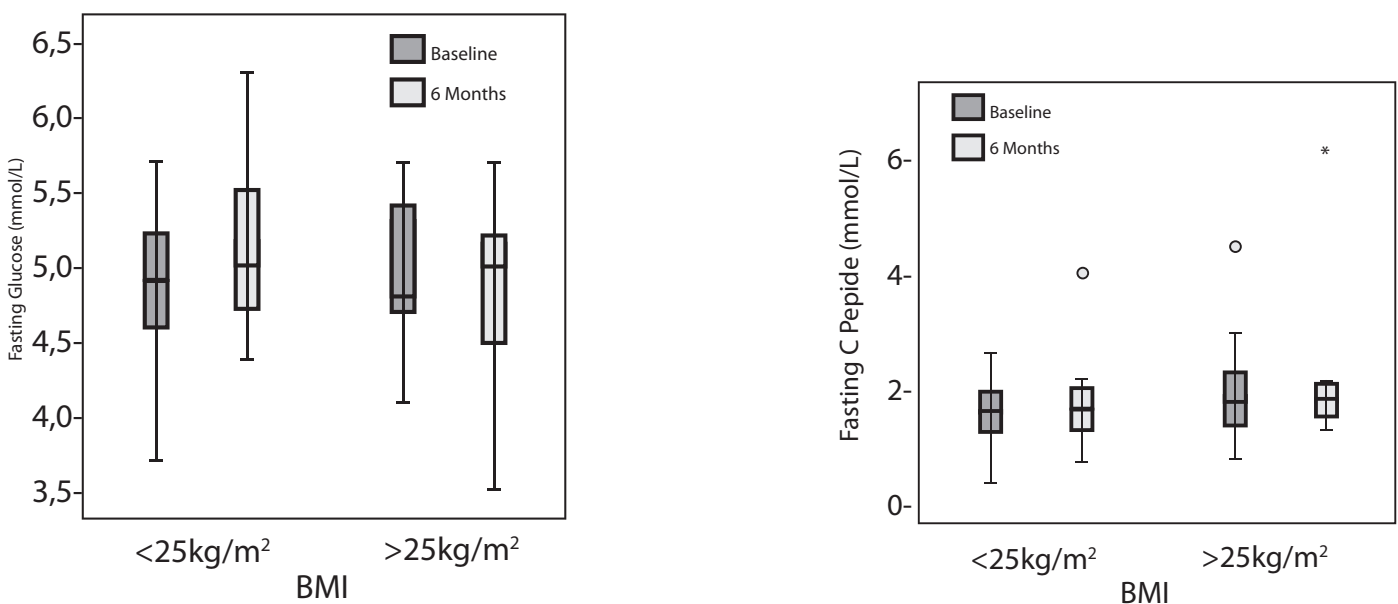

Fig. 2, 3. The comparison of fasting glucose and $\mathrm{C}$ peptide level among study subjects No significant association ( $\mathrm{p}>0.05)$
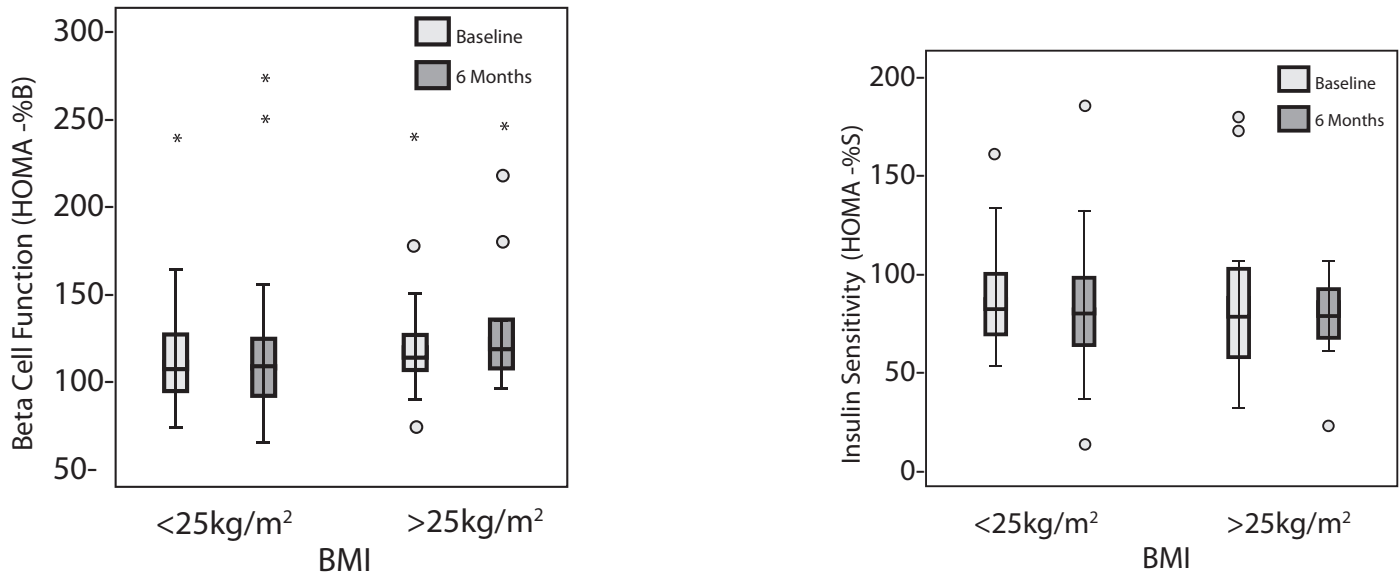

Fig. 4,5. The comparison of B Cell Function (HOMA-B \%) and insulin sensitivity among study subjects No significant association ( $\mathrm{p}>0.05)$

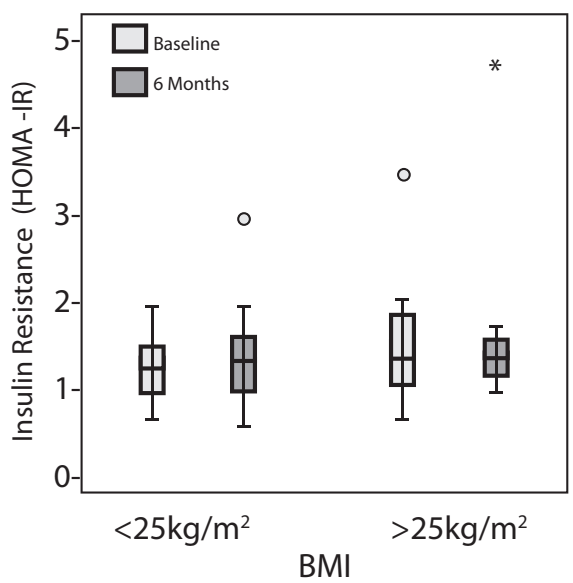

Fig. 6. The comparison of insulin resistance among study subjects

No significant association $(\mathrm{p}>0.05)$ 